\title{
Use of a low-cost infrared sensor for educational purpose: statistical analysis of results obtained by students
}

\author{
by M. Zamengo*, J. Morikawa*
}

*School of Materials and Chemical Technology, Tokyo Institute of Technology, Tokyo-to Meguro-ku Ookayama 2-12-1 S8-29, 152-8550, Japan, zamengo.m.aa@m.titech.ac.jp, morikawa.j.aa@m.titech.ac.jp

\begin{abstract}
A low-cost infrared (IR) camera was utilized for measurement of thermal diffusivity of three materials using the flash method by $3^{\text {rd }}$ grade bachelor students during one of their experiment class. Thermal diffusivity of the materials could be determined with good repeatability, despite the sources of errors originated mainly by measurement of thickness of specimens with caliper and noise of infrared signal. In this work, a statistical analysis of the data is presented and it confirmed the good quality of the measurements obtained using low cost instrumentation.
\end{abstract}

\section{Introduction}

In order to teach students some basic concepts of heat transfer properties, how to measure them and how to process the raw data, a low-cost infrared IR camera resulted a convenient educational tool. The IR camera was utilized for measuring thermal diffusivity of three different specimens made of polyamide (PA), polyoxymethylene (POM) and rubber by the flash method [1]. The results of experiments were analyzed statistically, in order to have an overall view of the quality of measurements obtained during a period of 2 years.

\section{Experimental apparatus and method}

A schematic of the apparatus and specimens is shown in Fig. 1 a) and b) respectively. The apparatus consists on an aluminium box containing the IR camera (compact thermal camera OWLIFT type-A, Infinitegra, Japan) and a support for the specimen. The flash lamp is positioned on the top. Raw data are recorded on PC and analysed using an MS Excel template, which facilitates the post processing of raw data and allows the determination of thermal diffusivity by numerical fitting using the least square method. As the specimens are relatively thick (about $5 \mathrm{~mm}$ ), two horizontal slits having different depth were engraved for reducing thickness, making it possible to detect stronger IR signal's intensity and measure thermal diffusivity on different positions of the specimen.
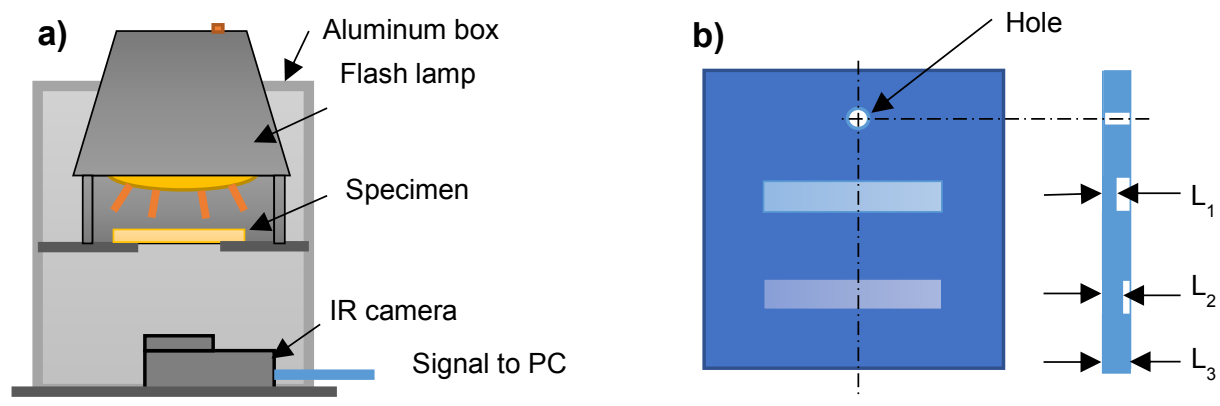

Fig. 1: a)Schematic of experimental apparatus used for the measurement of thermal diffusivity via the flash method; b) schematic of specimen object of measurements

The raw data of IR signal were normalized using Eq. (1), where $i(t)$ is the average intensity of IR signal on the regions corresponding to the slits, while $i_{\max }$ and $i_{\min }$ are the maximum and minimum intensities measured on the same regions, respectively:

$$
\theta(t)=\frac{i(t)-i_{\min }}{i_{\max }-i_{\min }}
$$

The fitting function is expressed by Eq. (2):

$$
\theta_{t h}(t)=1+2 \sum_{n=1}^{\infty}(-1)^{n} \exp \left(-n^{2} \pi^{2} \alpha t / L^{2}\right)
$$

where $\alpha$ is the thermal diffusivity, $t$ is time and $L$ is the thickness of the specimen in correspondence of the region of measurement. 


\section{Results and discussion}

The typical results of experiments are shown in Fig. 2 (PA specimen): they indicate that it was possible to detect the change of IR signal intensity and obtain good results from numerical fitting. The total average values of thermal diffusivity are reported in Table 1 (the number of data is 162,129 and 102, respectively for PA, POM and rubber). The results in Fig. 3 shows that the distribution of results can be described by a Gaussian type distribution in case of PA and $\mathrm{POM}$, while rubber deviates from it. The reason is attributed to non-homogeneous distribution of heat and errors which occurred during the measurements, especially the measurement of thickness on soft rubber.
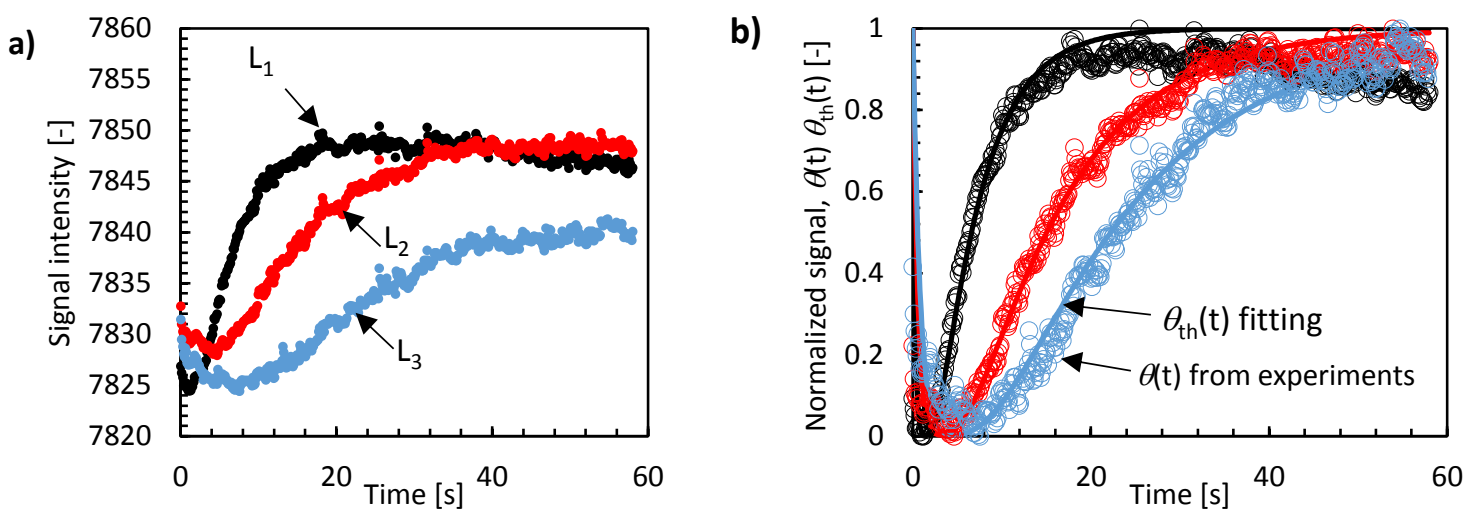

Fig. 2: a) Typical results of raw data of IR signal intensity measured for a PA specimen in correspondence of thickness $L_{1}, L_{2}$ and $L_{3}$; b) normalization of raw data and comparison with fitting curve
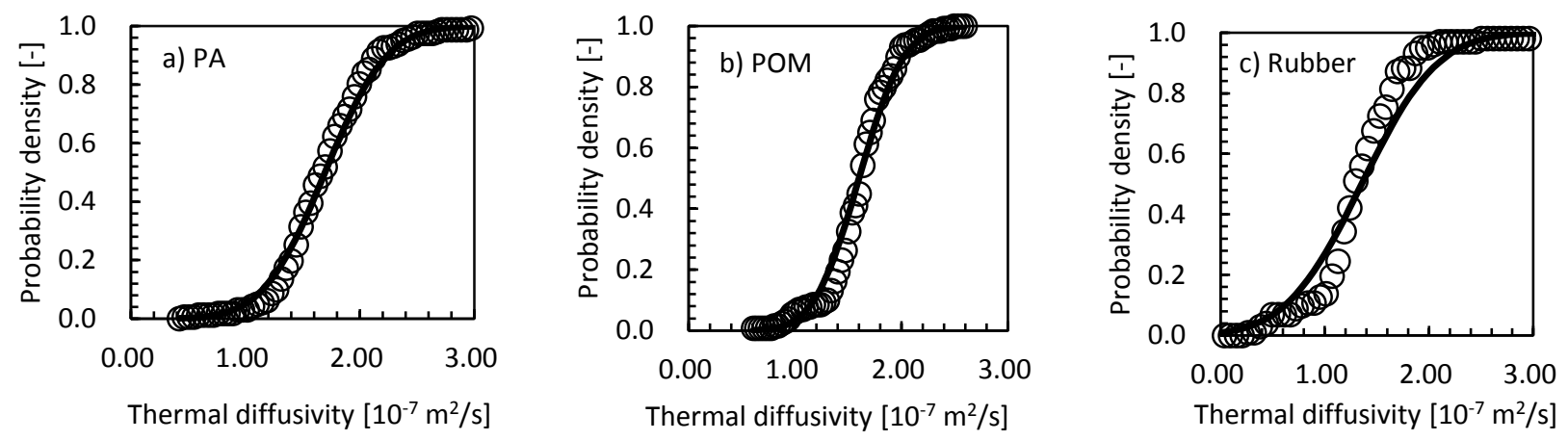

Fig. 3: Comparison of results distribution with Gaussian type distribution for a) $P A$, b) $P O M$ and c) rubber

Table 1. Average results of thermal diffusivity obtained by using the IR camera and flash method

\begin{tabular}{|l|l|}
\hline Material & Thermal diffusivity $\left[\mathrm{m}^{2} \mathrm{~s}^{-1}\right]$ \\
\hline PA (162 measurements) & $(1.70 \pm 0.42) \cdot 10^{-7}$ \\
\hline POM (129 measurements) & $(1.60 \pm 0.33) \cdot 10^{-7}$ \\
\hline Rubber (102 measurements) & $(1.35 \pm 0.57) \cdot 10^{-7}$ \\
\hline
\end{tabular}

\section{Conclusions}

Thermal diffusivity of PA, POM and rubber specimens was measured by using a low-cost IR camera. The results obtained follow a Gaussian distribution, except for rubber. The experiments showed a ratio standard deviation/average of $25 \%, 20 \%$ and $42 \%$ respectively for PA, POM and rubber. The wide spread of results around the average, especially for rubber, is likely related to non-homogeneous heat diffusion on the specimens and poor measurement of thickness.

\section{REFERENCES}

[1] W. J. Parker et al. Flash Method of Determining Thermal Diffusivity, Heat Capacity, and Thermal Conductivity, Journal of Applied Physics 32 (1961), p. 1679 